\title{
Ultrassonometria do calcâneo, densitometria óssea e morfometria vertebral em homens com idade acima de 60 anos
}

\author{
Quantitative ultrasound of the calcaneus, bone densitometry \\ and vertebral morphometry in men over the age of 60 years
}

Filipe Ramos Barra', André Ferreira Leite ${ }^{2}$, Paulo Tadeu de Souza², Fernando Borges Pereira ${ }^{3}$, Ana Patrícia de Paula ${ }^{4}$

\footnotetext{
1 Imagens Médicas de Brasília (IMEB), Brasilia, DF, Brasil ${ }^{2}$ Hospital Universitário de Brasilia, Brasília, DF, Brasil ${ }^{3}$ Programa de Pós-Graduação, Faculdade de Ciências da Saúde, Universidade de Brasília (UnB), Brasília, DF, Brasil ${ }^{4}$ Hospital de Base do Distrito Federal, SES-DF, Brasilia, DF, Brasil
}

\begin{abstract}
RESUMO
Objetivos: Comparar a ultrassonometria do calcâneo com a densitometria óssea na avaliação de fraturas vertebrais morfométricas em homens acima de 60 anos. Sujeitos e métodos: Foram analisados 96 homens com mais de 60 anos por meio de densitometria óssea da coluna, fêmur e rádio, radiografia lateral da coluna torácica e lombar e ultrassonometria do calcâneo. Resultados: Cinquenta e um por cento dos homens apresentaram osteoporose e fraturas vertebrais. Foi observada correlação entre índice de stiffness e T-score da ultrassonometria e as densidades minerais ósseas (DMO) de todos os sítios. Quanto à presença de fraturas, identificou-se correlação com a DMO do rádio ultradistal e $33 \%$. Por meio da curva ROC, observou-se acurácia da DMO do rádio UD na deteç̧ão de fraturas vertebrais. Conclusões: Nosso estudo mostrou correlação entre a ultrassonometria e a densitometria no diagnóstico de osteoporose em homens acima dos 60 anos. Também se identificou correlação entre fratura vertebral morfométrica e a DMO do rádio. Arq Bras Endocrinol Metab. 2012;56(6):370-5
\end{abstract}

\section{Descritores}

Osteoporose; fraturas vertebrais; fraturas por osteoporose; densitometria óssea

\begin{abstract}
Objectives: To compare calcaneal ultrasonometry and bone densitometry in the evaluation of morphometric vertebral fractures in men over 60 years of age. Subjects and methods: We studied 96 men over 60 years of age by means of bone densitometry of the spine, femur and radius, lateral radiograph of the thoracic and lumbar spine, and calcaneal ultrasonometry. Results: Fifty-one percent of men had osteoporosis and vertebral fractures. Correlation was found between ultrasonometry stiffness index, T-score and bone mineral density of the spine, femur and radius $(p<0.01)$. Regarding the presence of fractures, there was a correlation only with BMD of the ultradistal radius (UD) and radius $33 \%$. ROC curve showed accuracy only of UD radius BMD in detecting vertebral fractures. Conclusions Our study showed a correlation between osteoporosis diagnosis by ultrasonometry and densitometry in men over 60 years. It also showed a correlation between morphometric vertebral fracture and bone mineral density of the forearm. Arq Bras Endocrinol Metab. 2012;56(6):370-5
\end{abstract}

Keywords

Osteoporosis; vertebral fractures; osteoporotic fractures; bone densitometry
Correspondência para: SMN Ramos Barra conjunto C, sobreloja 15/16 70710-100 - Brasília, DF, Brasil

Recebido em 19/Dez/2011 Aceito em 27/Jul/2012

\section{INTRODUÇÃO}

$\Lambda \mathrm{s}$ fraturas por trauma mínimo são um importante Aproblema de saúde pública (1-3). A osteoporose em homens apresenta alto impacto social e econômico, com aumento da incidência nos últimos anos e alta mortalidade em decorrência das fraturas (4). A fratura vertebral 
é a mais comum e pode ser avaliada por análise morfométrica de radiografias laterais de coluna torácica e lombar e por análise com o software de avaliação de fratura vertebral contido em aparelhos de densitometria óssea $(5,6)$.

A densitometria óssea é o padrão-ouro para diagnosticar osteoporose, predizer risco de fratura, indicar início do tratamento e monitorá-lo (7-9), entretanto não é o único preditor de fraturas $(10,11)$. A densitometria óssea não deve ser usada isoladamente para predizer o risco de fratura.

A ultrassonometria óssea é um método de fácil execução, rápido, portátil, barato e livre de radiação ionizante (12), que, quando realizada com equipamentos validados, prediz risco de fratura em homens e mulheres idosos. O índice de stiffness é um dos quatro parâmetros validados para predizer fraturas por osteoporose e apresenta maior capacidade para predizer fratura que o coeficiente de atenuação do som (BUA) e a velocidade do som (SOS). O índice de stiffness é uma medida resultante de uma fórmula matemática que combina valores normatizados de SOS e BUA ( $\mathrm{SI}=0,67 \mathrm{X}$ BUA $+0,28$ X SOS - 420). Esse índice foi desenvolvido com o objetivo de melhorar a precisão do método (13-15).

Considerando a escassez de dados brasileiros, o presente estudo tem o objetivo de avaliar o resultado da densitometria e da ultrassonometria ósseas em pacientes com e sem fratura vertebral morfométrica.

\section{SUJEITOS E MÉTODOS}

Utilizou-se um banco de dados composto de 334 homens, atendidos pelo ambulatório da saúde do homem idoso, do Programa de Diagnóstico e Prevenção de Osteoporose da cidade de São Sebastião, DF. Para obtenção desses dados, os homens listados no cadastro de vacinação do centro de saúde de São Sebastião (DF) foram contatados pelo telefone ou pelos agentes comunitários para realização de projetos de pesquisas previamente aprovados pelo Comitê de Ética e Pesquisa (CEP) da Faculdade de Ciências da Saúde da Universidade de Brasília. Esta pesquisa foi encaminhada para avaliação do $\mathrm{CEP} / \mathrm{FS} / \mathrm{UnB}$, que considerou não ser necessária análise por se tratar de uso de banco de dados.

Foram incluídos os dados de todos os homens acima de 60 anos que concordaram em participar das pesquisas prévias e assinaram o termo de consentimento livre e esclarecido. Todos os pacientes foram inicialmente avaliados por questionário e encaminhados para consulta médica e exames laboratoriais, radiológicos e densi- tométricos no Hospital Universitário de Brasília. Foram selecionados aqueles que realizaram ultrassonometria e densitometria ósseas além de radiografias laterais de coluna torácica e lombar. Os dados de pacientes com diagnóstico de outras doenças osteometabólicas e em tratamento para osteoporose no momento da realização dos exames foram excluídos da análise. Todos os 96 homens incluídos no estudo apresentavam exames laboratoriais normais referentes às funções hepática, renal e gonadal. Nenhum homem apresentava fratura vertebral sintomática. Foi confirmada a presença de fratura não vertebral em quatro homens, distribuída quanto à localização em quadril, costela e duas fraturas de Colles.

A densitometria óssea por dupla emissão foi realizada utilizando o aparelho Lunar DPX-NT (General Eletrics, Estados Unidos). A aquisição das imagens para a avaliação da densidade mineral óssea considerou os seguintes sítios: coluna lombar, fềmur proximal direito e antebraço distal não dominante. A aquisição das imagens obedeceu às normas do fabricante. A análise densitométrica foi realizada por uma única médica habilitada pela Sociedade Brasileira de Densitometria Clínica (SBDens) de acordo com as normas da referida sociedade em concordância com a literatura internacional (16). O diagnóstico densitométrico baseou-se nos critérios da Organização Mundial de Saúde (17), sendo considerado o menor valor dos sítios analisados (L1-L4, colo femoral, fêmur total e rádio 33\% não dominante). Considerando que, segundo a National Osteoporosis Foundation (18), anteriormente ao desenvolvimento do FRAX, devia ser considerado tratamento medicamentoso em um paciente idoso com T-score densitométrico $\leq-2,0$, também foram apresentados dados quanto aos grupos de T-score $\leq \mathrm{e}>-2,0$.

A ultrassonometria óssea de calcâneo foi realizada no aparelho portátil Achilles Express (General Eletrics, Estados Unidos) utilizando o pé direito. Uma única técnica realizou todos os exames seguindo as normas do fabricante do aparelho. Os resultados disponíveis para análise eram índice de stiffness e T-score.

As fraturas vertebrais foram analisadas em radiografias laterais da coluna torácica e lombar. Foi realizada a avaliação semiquantitativa proposta por Genant e cols. (5). Neste método, realiza-se a marcação de cada corpo vertebral em seis pontos: dois anteriores, dois médios e dois posteriores. A altura anterior, média e posterior dos corpos vertebrais de T4 a L4 foi então medida e o diagnóstico de fratura baseou-se no grau de diminuição da altura vertebral. 
Os cálculos estatísticos foram realizados com o programa IBM SPSS para Windows versão 19.0, e os dados que caracterizam a amostra foram descritos com uso da média e do desvio-padrão. Após a verificação da normalidade dos dados por meio do teste de Kolmogorov-Smirnov, as correlações entre os fatores de risco mensurados e os resultados da ultrassonometria óssea foram testadas por meio de coeficientes de correlação de Pearson, com nível de significância. As diferenças entre as variáveis nos grupos com e sem fratura e nos grupos com diagnóstico densitométrico de $\mathrm{T}$-score $\leq \mathrm{e}$ $>-2,0$ foram testadas por meio do teste $t$ de Student.

A análise da curva ROC foi utilizada para determinação da validade da idade, do peso, da estatura, do índice de stiffness e da DMO da coluna, do fềmur total, do colo femoral, do rádio $33 \%$ e ultradistal visando ao diagnóstico de fratura vertebral, além da validade dos dados da ultrassonometria visando ao diagnóstico densitométrico de osteoporose e à baixa massa mineral óssea. Foi considerada uma área abaixo da curva ROC menor ou igual a 0,5 indicativa do não oferecimento de informação significativa do teste diagnóstico. Uma área entre 0,5 e 0,7 representa baixa acurácia; entre 0,7 e 0,9, moderada acurácia; e uma área igual a um indica a perfeição do teste.

\section{RESULTADOS}

Os dados da amostra relativos à idade, aos parâmetros antropométricos e aos hábitos de vida encontram-se descritos na tabela 1 . Tabagismo foi relatado por $25 \%$ dos homens, $8 \%$ eram etilistas e $72 \%$ consumiam café diariamente.

Tabela 1. Idade e parâmetros antropométricos da amostra estudada

\begin{tabular}{lc}
\hline & Média \pm DP \\
\hline Idade $(\mathrm{anos})$ & $68,31 \pm 5,772$ \\
Peso $(\mathrm{kg})$ & $67,65 \pm 11,28$ \\
Estatura $(\mathrm{m})$ & $1,63 \pm 0,061$ \\
IMC $\left(\mathrm{kg} / \mathrm{m}^{2}\right)$ & $25,4 \pm 3,43$ \\
\hline
\end{tabular}

Na tabela 2, apresentam-se os resultados da densitometria e ultrassonometria ósseas. Dos 96 homens avaliados, 49 (51\%) apresentaram diagnóstico densitométrico de osteoporose, $38(39,6 \%)$ foram considerados osteopênicos e apenas $9(9,4 \%)$ apresentaram densidade mineral óssea normal. Sessenta e seis pacientes $(68,7 \%)$ receberam diagnóstico de $\mathrm{T}$-score $\leq-2,0$.
Tabela 2. Resultados da densitometria e ultrassonometria ósseas

\begin{tabular}{lcccc}
\hline & Mínimo & Máximo & Média & $\begin{array}{c}\text { Desvio- } \\
\text {-padrão }\end{array}$ \\
\hline DM0 colo femoral $\left(\mathrm{g} / \mathrm{cm}^{2}\right)$ & 0,618 & 1,254 & 0,854 & 0,122 \\
T-score colo femoral & $-3,42$ & 1,43 & $-1,62$ & 0,97 \\
DM0 fêmur total $\left(\mathrm{g} / \mathrm{cm}^{2}\right)$ & 0,603 & 1,269 & 0,915 & 0,129 \\
T-score fêmur total & $-3,10$ & 1,30 & $-1,24$ & 0,92 \\
DMO L1-L4 $\left(\mathrm{g} / \mathrm{cm}^{2}\right)$ & 0,569 & 1,570 & 0,979 & 0,156 \\
T-score L1-L4 & $-4,74$ & 2,90 & $-1,89$ & 1,27 \\
DM0 rádio 33\% $\left(\mathrm{g} / \mathrm{cm}^{2}\right)$ & 0,497 & 0,893 & 0,719 & 0,084 \\
T-score rádio 33\% & $-3,80$ & 1,10 & $-1,06$ & 1,04 \\
DM0 rádio UD $\left(\mathrm{g} / \mathrm{cm}^{2}\right)$ & 0,244 & 0,524 & 0,371 & 0,069 \\
T-score rádio UD & $-4,40$ & 2,40 & $-1,27$ & 1,51 \\
T-score USO & $-3,10$ & 1,60 & $-1,09$ & 1,09 \\
Índice de stiffness & 50,00 & 126,00 & 82,80 & 17,96 \\
\hline
\end{tabular}

Não houve correlação entre os resultados da ultrassonometria óssea e a idade e os parâmetros antropométricos. Observou-se correlação significativa do valor do índice de stiffness e do T-score da ultrassonometria com os dados densitométricos de todos os sítios (Tabela 3). Ao se estratificarem os grupos, não houve diferença estatística entre os dados da ultrassonometria quanto à presença de T-score densitométrico $\leq-2,0$ nem quanto à presença de fraturas (Tabelas 4 e 5 ).

Foi obtida curva ROC para avaliar a sensibilidade e a especificidade dos parâmetros avaliados na detecção de fraturas vertebrais. Os dados são demonstrados na tabela 6 .

Considerando os grupos com presença ou ausência de fraturas morfométricas vertebrais, houve diferença significativa apenas quanto à densidade mineral óssea do rádio não dominante (Tabela 4).

Tabela 3. Correlação entre os resultados da densitometria e ultrassonometria ósseas

\begin{tabular}{|c|c|c|}
\hline Dados da densitometria & Índice de stiffness & T-score USO \\
\hline DMO colo femoral $\left(\mathrm{g} / \mathrm{cm}^{2}\right)$ & $0,266^{\star *}$ & $0,279^{* *}$ \\
\hline T-score colo femoral & $0,266^{\star *}$ & $0,280^{* *}$ \\
\hline DMO fêmur total $\left(\mathrm{g} / \mathrm{cm}^{2}\right)$ & $0,302^{\star \star}$ & $0,320^{\star *}$ \\
\hline T-score fêmur total & $0,298^{\star *}$ & $0,317^{\star \star}$ \\
\hline DMO L1-L4 (g/cm²) & $0,490^{\star *}$ & $0,453^{* *}$ \\
\hline T-score L1-L4 & $0,466^{\star *}$ & $0,430^{* *}$ \\
\hline DM0 rádio 33\% (g/cm²) & $0,287^{\star \star}$ & $0,279^{\star *}$ \\
\hline T-score rádio 33\% & $0,266^{\star \star}$ & $0,259^{*}$ \\
\hline DM0 rádio UD (g/cm²) & $0,418^{* *}$ & $0,421^{\star *}$ \\
\hline T-score rádio UD & $0,413^{\star *}$ & $0,416^{\star \star}$ \\
\hline
\end{tabular}

Correlação de Pearson, ${ }^{*} p<0,05 ;{ }^{* *} p<0,01$. 
Tabela 4. Distribuição dos dados de acordo com a presença ou ausência de fraturas

\begin{tabular}{|c|c|c|c|c|}
\hline & $\begin{array}{c}\text { Fraturas } \\
\text { vertebrais }\end{array}$ & $\mathbf{n}$ & Média & $\begin{array}{l}\text { Desvio- } \\
\text {-padrão }\end{array}$ \\
\hline \multirow[t]{2}{*}{ Idade (anos) } & Não & 49 & 67,61 & 5,37 \\
\hline & Sim & 47 & 69,04 & 6,13 \\
\hline \multirow[t]{2}{*}{ Peso (kg) } & Não & 49 & 68,87 & 12,03 \\
\hline & Sim & 47 & 66,37 & 10,44 \\
\hline \multirow[t]{2}{*}{ IMC $\left(\mathrm{kg} / \mathrm{m}^{2}\right)$} & Não & 49 & 25,53 & 3,31 \\
\hline & Sim & 47 & 25,24 & 3,58 \\
\hline \multirow[t]{2}{*}{ Altura (m) } & Não & 49 & 1,64 & 0,07 \\
\hline & Sim & 47 & 1,62 & 0,06 \\
\hline \multirow[t]{2}{*}{ DMO colo femoral $\left(\mathrm{g} / \mathrm{cm}^{2}\right)$} & Não & 48 & 0,87 & 0,13 \\
\hline & $\operatorname{Sim}$ & 46 & 0,84 & 0,11 \\
\hline \multirow[t]{2}{*}{ T-score colo femoral } & Não & 48 & $-1,55$ & 1,05 \\
\hline & Sim & 46 & $-1,70$ & 0,90 \\
\hline \multirow[t]{2}{*}{ DM0 quadril total $\left(\mathrm{g} / \mathrm{cm}^{2}\right)$} & Não & 48 & 0,93 & 0,14 \\
\hline & Sim & 46 & 0,90 & 0,12 \\
\hline \multirow[t]{2}{*}{ T-score quadril total } & Não & 48 & $-1,13$ & 1,00 \\
\hline & Sim & 46 & $-1,37$ & 0,82 \\
\hline \multirow[t]{2}{*}{ DMO L1-L4 (g/cm²) } & Não & 47 & 0,98 & 0,15 \\
\hline & $\operatorname{Sim}$ & 46 & 0,98 & 0,16 \\
\hline \multirow[t]{2}{*}{ T-score L1-L4 } & Não & 47 & $-1,90$ & 1,25 \\
\hline & Sim & 46 & $-1,88$ & 1,31 \\
\hline \multirow[t]{2}{*}{ DM0 rádio UD $\left(\mathrm{g} / \mathrm{cm}^{2}\right)$} & Não & 45 & $0,39^{*}$ & 0,06 \\
\hline & Sim & 46 & $0,35^{*}$ & 0,06 \\
\hline \multirow[t]{2}{*}{ T-score rádio UD } & Não & 45 & $-0,79^{*}$ & 1,53 \\
\hline & Sim & 46 & $-1,75^{\star}$ & 1,36 \\
\hline \multirow[t]{2}{*}{ T-score USO } & Não & 49 & $-0,90$ & 1,06 \\
\hline & Sim & 47 & $-1,30$ & 1,11 \\
\hline \multirow[t]{2}{*}{ Índice de stiffness } & Não & 49 & 85,63 & 16,81 \\
\hline & Sim & 47 & 79,85 & 18,83 \\
\hline
\end{tabular}

Teste $t$ de Student, ${ }^{*} p<0,05$.

Tabela 5. Distribuição dos dados da ultrassonometria de acordo com resultado densitométrico

\begin{tabular}{lcc}
\hline Dados da US0 & $\begin{array}{c}\text { T-score } \leq \mathbf{- 2 , 0} \\
(\bar{X} \pm \mathbf{D P}) \mathbf{n}=\mathbf{6 6}\end{array}$ & $\begin{array}{c}\text { T-score } \mathbf{~ - 2 , 0} \\
(\bar{X} \pm \mathbf{D P}) \mathbf{n}=\mathbf{3 0}\end{array}$ \\
\hline Índice de stiffness & $78,878 \pm 17,060$ & $91,433 \pm 17,123$ \\
T-score USO & $-1,343 \pm 1,020$ & $-0,543 \pm 1,066$ \\
\hline
\end{tabular}

Teste $t$ de Student. Não houve diferença significativa entre os dados.

Apenas quatro homens referiram história prévia de outras fraturas não vertebrais e todos estavam no grupo com fratura morfométrica vertebral.
Tabela 6. Dados da curva ROC dos parâmetros avaliados nos 96 homens estudados em relação à presença de alterações morfométricas

\begin{tabular}{lcc}
\hline Dados avaliados & $\begin{array}{c}\text { Área abaixo da curva } \\
\text { (intervalo de confiança de 95\%) }\end{array}$ & p \\
\hline Idade (anos) & $0,564(0,430-0,662)$ & 0,276 \\
Peso (kg) & $0,5460,430-0,662)$ & 0,437 \\
IMC (kg/m²) & $0,535(0,418-0,651)$ & 0,560 \\
DM0 colo femoral (g/cm²) & $0,547(0,430-0,665)$ & 0,432 \\
T-score colo femoral & $0,549(0,426-0,67)$ & 0,430 \\
DM0 fêmur total $\left(\mathrm{g} / \mathrm{cm}^{2}\right)$ & $0,548(0,431-0,665)$ & 0,423 \\
T-score fêmur total & $0,556(0,434-0,678)$ & 0,370 \\
DM0 L1-L4 (g/cm²) & $0,497(0,378-0,615)$ & 0,954 \\
T-score L1-L4 & $0,487(0,365-0,610)$ & 0,894 \\
DM0 rádio 33\% (g/cm²) & $0,603(0,486-0,720)$ & 0,091 \\
T-score rádio 33\% & $0,596(0,477-0,716)$ & 0,121 \\
DM0 rádio UD (g/cm²) & $0,667(0,556-0,778)$ & $0,006^{*}$ \\
T-score rádio UD & $0,643(0,528-0,759)$ & $0,021^{*}$ \\
Índice de stiffness & $0,605(0,491-0,719)$ & 0,076 \\
T-score US0 & $0,607(0,488-0,726)$ & 0,086 \\
\hline
\end{tabular}

${ }^{*} p<0,05$

\section{DISCUSSÃO}

Neste estudo, ficou demonstrada correlação positiva entre as densidades minerais óssea avaliadas e o peso, a estatura e o índice de massa corporal. Houve correlação negativa entre a idade e as densidades minerais ósseas do colo femoral, do quadril total, do rádio $33 \%$ e do rádio ultradistal. Resultados semelhantes foram encontrados por estudo de Mulleman e cols. (19). Não houve correlação entre o índice de stiffness, o T-score do índice do stiffness e a idade e a estatura, corroborando com os resultados encontrados em outro estudo brasileiro (20).

Ficou demonstrado que existe associação entre os resultados obtidos da ultrassometria do calcâneo e da densitometria óssea para o diagnóstico de osteoporose, corroborando com sua utilização na identificação de pacientes com massa óssea reduzida. Estudos prévios mostraram resultados semelhantes em mulheres e homens (20-23).

Pacientes com T-score densitométrico menor ou igual a -2,0 tinham menor valor do índice de stiffness e menor T-score, sugerindo que um menor valor do índice de stiffiness em uma população de risco auxilia os clínicos a suspeitar do diagnóstico de osteoporose e atuar na prevenção de risco de fraturas. Não foi encontrada significância estatística, porém resultado signifi- 
cante foi encontrado por outros autores ao avaliarem 102 pacientes (19).

Quanto à presença de fratura morfométrica vertebral, não houve correlação em relação à idade, ao peso, ao IMC, às densidades minerais ósseas de coluna lombar e do colo femoral e quadril total. Entretanto, houve diferença significativa, entre os grupos com e sem fratura, quanto à densidade mineral óssea do rádio ultradistal e 33\%. Esse dado foi também evidenciado com a obtenção da curva ROC, em que o único parâmetro avaliado que apresentou acurácia baixa, porém significante para a detecção de fraturas, foi a DMO do rádio ultradistal. Isso também foi evidenciado por Pasco e cols. (24) ao avaliar uma amostra composta por 555 homens.

Considerando que a diferença encontrada, entre os grupos classificados quanto à presença ou à ausência de fraturas morfométricas, foi apenas em relação à densidade mineral óssea de rádio, é importante ressaltar que a presença de alterações degenerativas em coluna lombar e em coxofemoral poderia ser responsável por um falso aumento das densidades minerais ósseas nesses sítios. Esse dado reforça a importância da avaliação densitométrica do antebraço distal, o que muitas vezes é deixado de lado na prática diária.

Ainda quanto à fratura vertebral, não foi detectada diferença quanto ao valor de índice de stiffness e do T-score relativo. Apesar de não ser estatisticamente significante, a média do T-score encontrada nos homens com fratura $(-1,3)$ é semelhante à demonstrada em um estudo chinês que avaliou 9.352 homens e mulheres quanto ao risco de fraturas (13). Houve tendência para um menor valor do índice de stiffness naqueles pacientes do grupo com fratura morfométrica e provavelmente com um número de pacientes maior poderia ser encontrada diferença, o que corroboraria o estudo de Frediani e cols. (25).

Outros estudos demonstraram que a ultrassonometria óssea pode predizer risco de fratura de quadril e não vertebral em homens e mulheres $(14,15,25,26)$. Neste estudo, em decorrência do pequeno número dessas fraturas, não foi possível fazer essa análise.

Neste estudo, a ultrassonometria óssea de calcâneo não detectou diferença entre os grupos de homens com e sem fratura vertebral morfométrica. Essa diferença foi observada apenas quanto aos resultados da densitometria de antebraço distal não dominante.

Declaração: os autores declaram não haver conflitos de interesse científico neste estudo.

\section{REFERÊNCIAS}

1. Cooper C, Campion G, Melton LJ, 3rd. Hip fractures in the elderly: a world-wide projection. Osteoporos Int. 1992;2(6):285-9.

2. Melton LJ, 3rd, Lane AW, Cooper C, Eastell R, O'Fallon WM, Riggs BL. Prevalence and incidence of vertebral deformities. Osteoporos Int. 1993;3(3):113-9.

3. O'Neill TW, Felsenberg D, Varlow J, Cooper C, Kanis JA, Silman AJ. The prevalence of vertebral deformity in European men and women: the European Vertebral Osteoporosis Study. J Bone Miner Res. 1996;11(7):1010-8.

4. Al Attia $\mathrm{H}$, Adams B. Osteoporosis in men: are we referring enough for DXA and how? Clin Rheumatol. 2007;26(7):1123-6.

5. Genant HK, Wu CY, van Kuijk C, Nevitt MC. Vertebral fracture assessment using a semiquantitative technique. J Bone Miner Res. 1993;8(9):1137-48.

6. Diacinti D, Guglielmi G. Vertebral morphometry. Radiol Clin North Am. 2010;48(3):561-75.

7. Lochmuller EM, Muller R, Kuhn V, Lill CA, Eckstein F. Can novel clinical densitometric techniques replace or improve DXA in predicting bone strength in osteoporosis at the hip and other skeletal sites? J Bone Miner Res. 2003;18(5):906-12.

8. Miller PD, Zapalowski C, Kulak CA, Bilezikian JP. Bone densitometry: the best way to detect osteoporosis and to monitor therapy. J Clin Endocrinol Metab. 1999;84(6):1867-71.

9. Kanis JA, Johnell O. Requirements for DXA for the management of osteoporosis in Europe. Osteoporos Int. 2005;16(3):229-38.

10. Costa-Paiva L, Horoviz A, Santos A, Fonsechi-Carvasan G, Pinto-Neto A. Prevalência da osteoporose em mulheres na pósmenopausa e associação com fatores clínicos e reprodutivos. RBGO. 2003;25(7):507-12.

11. Taguchi A, SueiY, Sanada M, Ohtsuka M, Nakamoto T, Sumida H, et al. Validation of dental panoramic radiography measures for identifying postmenopausal women with spinal osteoporosis. AJR Am J Roentgenol. 2004;183(6):1755-60.

12. Brunader $R$, Shelton $D K$. Radiologic bone assessment in the evaluation of osteoporosis. Am Fam Physician. 2002;65(7):1357-64.

13. Liu JM, Ma LY, Bi YF, Xu Y, Huang Y, Xu M, et al. A populationbased study examining calcaneus quantitative ultrasound and its optimal cut-points to discriminate osteoporotic fractures among 9352 Chinese women and men. J Clin Endocrinol Metab. 2012;97(3):800-9.

14. Bauer DC, Ewing SK, Cauley JA, Ensrud KE, Cummings SR, Orwoll ES. Quantitative ultrasound predicts hip and non-spine fracture in men: the MrOS study. Osteoporos Int. 2007;18(6):771-7.

15. Diez-Perez A, Gonzalez-Macias J, Marin F, Abizanda M, Alvarez R, Gimeno A, et al. Prediction of absolute risk of non-spinal fractures using clinical risk factors and heel quantitative ultrasound. Osteoporos Int. 2007;18(5):629-39.

16. NIH. Consensus Statement. Osteoporosis prevention, diagnosis, and therapy. JAMA. 2001;285(6):785-95.

17. Currey J. Role of collagen and other organics in the mechanical properties of bone. Osteoporos Int. 2003;14 Suppl 5:29-36.

18. National Osteoporosis Foundation. Physician's Guide to Prevention and Treatment of Osteoporosis. [online] 1999. Disponível em: http://www.nof.org/professionals/clinical-guidelines. Acesso em: 15 maio, 2000.

19. Mulleman D, Legroux-Gerot I, Duquesnoy B, Marchandise $X$, Delcambre B, Cortet B. Quantitative ultrasound of bone in male osteoporosis. Osteoporos Int. 2002;13(5):388-93.

20. Oliveira PP, Marinheiro LPF, Wender MCO, Roisenberg F. O uso da ultrassonometria óssea de calcâneo Sonost 2000 para rastreamento de populações em risco para fraturas vertebrais. Rev Bras Med. 2011;68(10):4. 
21. Trimpou P, Bosaeus I, Bengtsson B-A, Landin-Wilhelmsen K. High correlation between quantitative ultrasound and DXA during 7 years of follow-up. Eur J Radiol. 2010;73(2):360-4.

22. Castro ML, Brandão C, Yassuda MY, Martin LNC, Vieira JGH. Correlação entre ultrassonometria quantitativa de calcâneo e densitometria óssea duo-energética de coluna e fêmur na avaliação óssea. Arq Bras Endocrinol Metab. 1999;43:205-9.

23. Schneider J, Bundschuh B, Spath C, Landkammer C, Muller H, Sommer $U$, et al. Discrimination of patients with and without vertebral fractures as measured by ultrasound and DXA osteodensitometry. CalcifTissue Int. 2004;74(3):246-54.
24. Pasco JA, Henry MJ, Korn S, Nicholson GC, Kotowicz MA. Morphometric vertebral fractures of the lower thoracic and lumbar spine, physical function and quality of life in men. Osteoporos Int. 2009;20(5):787-92.

25. Frediani B, Acciai C, Falsetti P, Baldi F, Filippou G, Siagkri C, et al. Calcaneus ultrasonometry and dual-energy $\mathrm{X}$-ray absorptiometry for the evaluation of vertebral fracture risk. CalcifTissue Int. 2006;79(4):223-9.

26. KwokT, Khoo CC, Leung J, KwokA, Qin L, Woo J, et al. Predictive values of calcaneal quantitative ultrasound and dual energy $X$ ray absorptiometry for non-vertebral fracture in older men: results from the MrOS study (Hong Kong). Osteoporos Int. 2012;23(3):1001-6. 